\title{
Monitoring of the cerebral tissue saturation in anemia of extremely preterm infants
}

\author{
Mihaela Demetrian, Andreea Avramescu, Vlad Dima, Roxana Iliescu \\ Filantropia Clinical Hospital of Obstetrics and Gynecology, Bucharest, Romania
}

\begin{abstract}
Objectives. In this study we investigated if the cerebral oxygenation measurements before and after the packed red blood cell transfusions (PRBC) can answer the question: was transfusion beneficial? Can the measurements derived from NIRS (near infrared spectroscopy) be useful for the identification of more objective criteria for the transfusion guidelines?

Patients and methods. This is a prospective observational study, performed in the period July 2017 - March 2018 , in the 3th neonatal intensive care unit. It enrolled 44 preterm infants, with a weight $\leq 1250 \mathrm{~g}$ and gestational age $\leq 30$ weeks, randomized by the PRBC transfusion in transfused group $(n=29)$ and non-transfused group $(n=15)$. The preterm infants that needed transfusion were NIRS monitored before (continuous monitoring $24 \mathrm{~h}$ ), during and $24 \mathrm{~h}$ after the transfusion. The non-transfused patients were also monitored with cerebral regional pulse-oximetry at postnatal age, and under clinical conditions similar to the transfused group.

Results. The values of the cerebral and systemic oximetry were comparable for the two groups when comparing the results before transfusion. Regarding the effects of transfusions, results showed a significant increase of cerebral tissue oxygenation ( $\mathrm{CrSO} 2$ ) even during the 4 hours of transfusion, an effect maintained 24 hours following transfusion: postransfusional mean $\mathrm{CrSO} 2=80 \pm 2, \mathrm{p}$ value 0.019 . Moreover, the values of the fractional cerebral tissue oxygen extraction begin to decrease during transfusion, and they remain low for the next $24 \mathrm{~h}$ as well $0.25 \pm 0.05$ vs $0.15 \pm 0.02-p$ value $<0.013$.

Conclusions. PRBC transfusion in clinically stable very low birth weight preterm leads to the transient increase of $\mathrm{CrSO} 2$ and transient decrease of FTOE. Our data support the use of measurements derived from NIRS (FTOE) for the identification of the sub-clinical imbalance in $\mathrm{O} 2$ delivery and consumption as objective measurement in anemic preterm infants.
\end{abstract}

Keywords: preterm infant anemia, transfusion, cerebral tissue saturation

\section{INTRODUCTION}

The last few years, the measurement of tissue regional oxygenation ( $\mathrm{rSO} 2)$ through near-infrared spectroscopy (NIRS) became commonly used in the neonatal intensive care units. NIRS has been used in several clinical situations in order to evaluate the cerebral oxygenation of preterm infants. There are studies that used cerebral regional saturation $\mathrm{CrSO} 2$ as biomarker for the need of $\mathrm{PRBC}$ transfusions, as well as cerebral oxygenation response after transfusion (1-3). An improvement in $\mathrm{CrSO} 2$ and a reduction of fractional cerebral tissue oxygen extraction - C-FTOE have been reported after PRBC transfusions. Some investigators reported a lack of correlation in pre-transfusion hematocrit with $\mathrm{CrSO} 2$, suggesting that hematocrit level is a poor predictor of tissue oxygenation. Both, cerebral regional and peripheral oxygen satu- ration $(\mathrm{CrSO} 2, \mathrm{prSO} 2)$, decrease when the oxygen transport capacity is compromised and increase during blood transfusion (4). Some authors investigated the relationship between the number of hypoxic incidents (HI) and packed red cell transfusion (PRBC), following the hypothesis that, if more hemoglobin is available, the oxygen transport is more effective, and the hypoxic episodes can be prevented $(5,6)$. A significant improvement in $\mathrm{CrSO} 2$, prSO2 perfusion and anemia symptoms was described after PRBC transfusion in preterm infants with $\mathrm{CrSO} 2<55 \%$ compared to preterm infants with crSO $2>55 \%$ (7-9). In this study we investigated whether NIRS measurements before and after PRBC transfusions can answer the questions: Was transfusion beneficial? NIRS derived measurements may be useful in PRBC transfusion guidelines? 
We hypothesized that measurement of cerebral saturation $(\mathrm{CrSO} 2)$ with NIRS is possible to select preterm infants that will have the greatest clinical benefit after PRBC transfusion. $\mathrm{CrSO} 2$ monitoring could detect the subclinical signs of the imbalance in $\mathrm{O} 2$ delivery and consumption (i.e. increased FTOE) that cannot be detected using conventional monitoring (e.g. physical examination, vital signs, pulse oximetry, hematocrit). In non-transfused preterm infants, monitored at an equivalent postnatal age, we should obtain NIRS signals comparable to the normal age-reference values for, allowing us to assign changes to the transfusion event.

\section{MATERIALS AND METHODS}

This prospective, observational, and non-interventional study was conducted in tertiary level neonatal intensive care between July 2017 and March 2018. The NIRS protocol involved filling observer monitoring data for preterm infants with high risk of anemia and PRBC transfusion. The inclusion criteria focused on preterm infants with $\mathrm{GA} \leq 30$ weeks and birth weight $\leq 1,250$ grams. Neonates weighing $<500$ grams, major congenital / chromosomal abnormalities, Apgar score $<3$ at 5 minutes, intraventricular hemorrhage grades 3-4 were excluded. The patients were enrolled within the first 72 hours after birth and were continuously monitored during hospitalization. The monitoring included: continuous cardio-respiratory monitoring and conventional pulse oximetry; weekly hematological assessment: hemoglobin, hematocrit, red blood cell indices, sideremia; caloric and anthropometric curves. Prior to and after transfusion, acid base balance was monitored from the blood gas analysis (BGA), $\mathrm{pH}$ and serum lactate were extracted for all preterm infants in the study.

Transfused group were NIRS monitored - cerebral regional oxygenation assessment method pretransfusion (continuous monitoring $24 \mathrm{~h}$ ), during transfusion, and $24 \mathrm{~h}$ after transfusion. Non-transfused patients were also monitored with cerebral regional pulse oximetry when they were respiratory and hemodynamically stable, with complete enteral feeding, weight gaining, at a postnatal age and under clinical conditions similar to transfused group. Monitoring $\mathrm{CrSO} 2$ was achieved using the cerebral / somatic FORE SIGHT ELITE oxymeter from CAS Medical Systems INC. In pediatric studies using this device, the accuracy of cerebral site with small sensor was $-0.01 \pm 5.38 \%$ and $0.03 \pm 5.69 \%$ for non-cerebral sites (10). This device reports the regional oxygenation status of the venous, arterial, and capillary hemoglobin sources, at a ration of approximately 75: 20:5, expressed as percentage of oxygenated hemoglobin to total hemoglobin. The NIRS monitoring sensors were located cross-sectional along the forehead. Absolute variables measured by NIRS during monitoring: cerebral regional saturation: $\mathrm{CrSO} 2$ and fractional cerebral tissue oxygen extraction: C-FTOE. CrSO2 regular reference range for preterm infants varies between $55 \%$ and $85 \%$, depending on factors such as the type of the device, clinical condition, and postnatal age. There are studies that showed a significant negative correlation between $\mathrm{CrSO} 2$ and the gestational age (r 1/4 0.77; $\mathrm{P}<0.001$ ). The highest level of $\mathrm{CrSO} 2$ and the lowest C-FTOE were found between weeks 30 and 33; subsequently, $\mathrm{CrSO} 2$ decreased progressively reaching a minimum value at weeks 3839 while cerebral tissue oxygen extraction (CFTOE) increased. In these studies, $\mathrm{CrSO} 2$ was also significantly correlated with heart rate, respiratory rate, and pulseoximetry ( $\mathrm{SaO} 2)$ values (r 1/4 0.65; $\mathrm{P}<0.001)$ (11-13). In our study we set values $71 \pm 7 \%$ as normal reference range for the preterm infant under 32 weeks and over 7 days (14). All patients were treated according to predefined strategies of ventilation, fluids, electrolytes and nutrition protocols. Screening echocardiography was performed for all patients on 3-5 days of postnatal life, and those subjects with hemodynamic significant PDA were treated with ibuprofen.

\section{Packed red cell (PRBC) transfusions}

They were administered according to hemoglobin and hematocrit values, but related to postnatal age, respiratory status, and associated clinical signs. Patients who received PRBC transfusions for hemorrhagic events (e.g. intraventricular hemorrhage) were not included in this analysis as the study is focused on detecting changes in cerebral oxygenation parameters in stable patients.

\section{Data collection and analysis}

Demographic data were extracted from patient's diagrams, along with NIRS measurements. The results obtained from the blood collected at 7 days during routine tests were used.

Non-transfused subjects were NIRS monitored for 12 hours at approximately 35 postnatal days representing the median postnatal age when the first transfusion was performed for transfused group.

Statistical analysis - Within the transfused group, pre- and post-transfusion analyzes were per- 
formed using t-Student for single data points and repeated ANOVA measurements for continuous measurements; $\mathrm{P}<0.05$ was used to assign the statistical significance for all analyses. In order to analyze the results of cerebral oxygenation pre-, during-, and post-transfusion, the Kruskal Wallis test and the Mann Whitney U test were used to calculate the magnitude of the effect of transfusion on CrSo2 an C-FTOE (effect size).

\section{RESULTS}

Initially there were 57 newborns between July 1 and March 31 with gestation age $\leq 30$ weeks and weight between 500 and 1,250 g but only 44 (77\%) met the eligibility criteria and had completed the monitoring protocol. The reasons for non-inclusion were: refusal of consent $(n=2)$, intraventricular hemorrhage $>$ gr. $3(n=3)$, death $(n=3)$ and unavailability of the equipment $(n=5)$. Selection of the subjects led to 44 stable, clinically predictable newborns. Of these, 29 preterm infants (66\%) were received at least one transfusion during hospitalization, and the entire group received a total of 57 transfusions, the median transfusions / baby being of $1.9 \pm 0.3$. The comparison group $(n=15)$ of nontransfused newborns had a higher gestational age $(28.5 / 26.2$ weeks), a higher weight at birth $1154 \pm 195 / 941 \pm 227 \mathrm{~g}$, less pregnancy pathology and a lower probability of intubation at birth, compared to transfused subjects ( $p<" 0.05)$. The hemoglobin and hematocrit values associated with clinical signs of anemia $(59 \%, \mathrm{n}=17)$ were the main reasons for PRBC transfusion. In the transfused group, the first transfusion was performed on the $35^{\text {th }}$ day $(35.8 \pm 18.3 ; 10-72)$ therefore between the two groups the hematological values of the fifth postnatal week were compared. There were no significant differences between hemoglobin, hematocrit, and red blood cell indices.

Results of the cerebral regional oxygenation (NIRS) - Cerebral and systemic oximetry values were comparable for both groups when comparing the results before the transfusion and they corresponded to the normal reference values for preterm infants under 32 weeks and over 7 days of life - Table 1 (14). No statistically significant changes in cerebral saturation - $\mathrm{CrSO} 2$ or C-FTOE in the nontransfused group at a postnatal equivalent age and over a 12-hour observation period.Concerning the effect of transfusions, the results showed a significant increase in cerebral tissue oxygenation (CrSO2) even during the 4 hours of transfusion monitoring, $73 \pm 2$ vs $75 \pm 6$, which is maintained for 24 hours post-transfusion: mean $\mathrm{CrSO} 2$ post-transfusion $=80 \pm 2$, p value 0.019 . Also, cerebral fractional oxygen extraction values begin to decrease during transfusion, and remain low over the next 24 hours $0.25 \pm 0.05$ vs $0.15 \pm 0.02$ - $p$ value $<0.013$ (Table 2, Fig. 1 and 2). No significant changes in perfusion index and serum lactate in two groups pre or post-transfusion were observed.

TABLE 1. Comparative results on cerebral oxygen monitoring before transfusion

\begin{tabular}{|l|c|c|c|}
\hline \multicolumn{1}{|c|}{ Variable* } & $\begin{array}{c}\text { Transfused } \\
(\mathbf{n = 2 9 )}\end{array}$ & $\begin{array}{c}\text { Nontransfused } \\
(\mathbf{n}=\mathbf{1 5})\end{array}$ & P value \\
\hline spO2 & $94 \pm 3$ & $93 \pm 5$ & 0.98 \\
\hline $\mathrm{CrSO2}$ & $73 \pm 2.6$ & $72 \pm 3.6$ & 0.26 \\
\hline $\mathrm{C}-\mathrm{FTOE}$ & $0.25 \pm 0.05$ & $0.23 \pm 0.02$ & 0.83 \\
\hline Seric Lactat & $1.28 \pm 0.2$ & $1.2 \pm 0.1$ & 0.61 \\
\hline Perfusion Index & $1.2 \pm 0.3$ & $1.3 \pm 0.5$ & 0.5 \\
\hline pH (median $\pm \mathrm{DS})$ & $7.33 \pm 0.06$ & $7.31 \pm 0.03$ & 0.25 \\
\hline
\end{tabular}

*(median $\pm \mathrm{DS}) \mathrm{CrSO} 2$ = cerebral regional oxygen saturation; $\mathrm{C}$-FTOE = cerebral fractional tissular oxygen extraction

\section{DISCUSSIONS}

PRBC transfusions in respiratory and hemodynamically stable VLBW preterm infants increased cerebral regional saturation $(\mathrm{CrSO} 2)$ and reduced fractional cerebral tissue oxygen extraction (CFTOE), as expected in the presence of anemia $(2,8,9)$. In transfused subjects, were not observed statistically significant changes in conventional

TABLE 2. Transfusions effect on cerebral oxygen saturation and cerebral oxygen delivery **

\begin{tabular}{|c|c|c|c|c|}
\hline Variable* & $\begin{array}{c}\text { Pretransion } \\
(24 \mathrm{~h})\end{array}$ & $\begin{array}{l}\text { During transfusion } \\
\qquad(4 \mathrm{~h})\end{array}$ & $\begin{array}{l}\text { Post-transfusion } \\
\text { (24 h) }\end{array}$ & $P$ value \\
\hline $\mathrm{SpO} 2(\%)$ & $94 \pm 3 / 94.78$ & $94 \pm 4 / 94.52$ & $94 \pm 2 / 94.71$ & .82 \\
\hline $\mathrm{CrSO} 2$ & $73 \pm 2 / 72.66$ & $75 \pm 1.5 / 74.94$ & $80 \pm 2 / 79.74$ & 0.0019 \\
\hline C-FTOE & $0.25 \pm 0.05 / 0.23$ & $0.19 \pm 0.04 / 0.21$ & $0.15 \pm 0.02 / 0.15$ & 0.0013 \\
\hline Seric lactat & $1.28 \pm 0.2$ & - & $1.4 \pm 0.3$ & 0.5 \\
\hline $\begin{array}{l}\text { Index de perfuzie } \\
\text { sulară }\end{array}$ & $1.2 \pm 0.3$ & - & $1.1 \pm 0.3$ & 0.8 \\
\hline $\mathrm{pH}$ (median $\pm \mathrm{DS})$ & $7.33 \pm 0.06$ & - & $7.30 \pm 0.03$ & 0.63 \\
\hline
\end{tabular}

*median \pm DS; $\mathrm{CrSO} 2$ = cerebral regional oxygen saturation; C-FTOE = cerebral fractional tissular oxygen extraction. $\mathrm{p}<0.05$ for all $\mathrm{CrSO} 2$ and FTOE pre-post calculated $* *$ (Kruskal Wallis Test) 


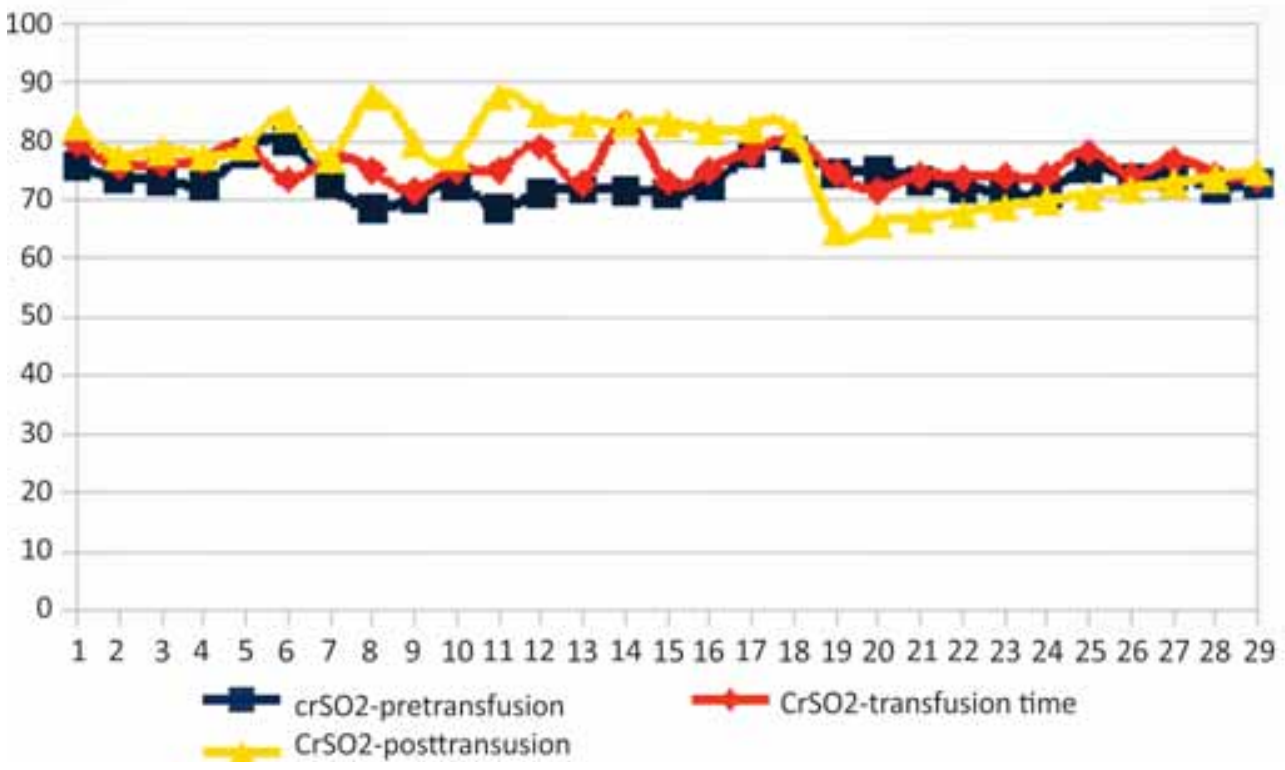

FIGURE 1. Effect of transfusion on cerebral oxygenation (CrSO2 curves)

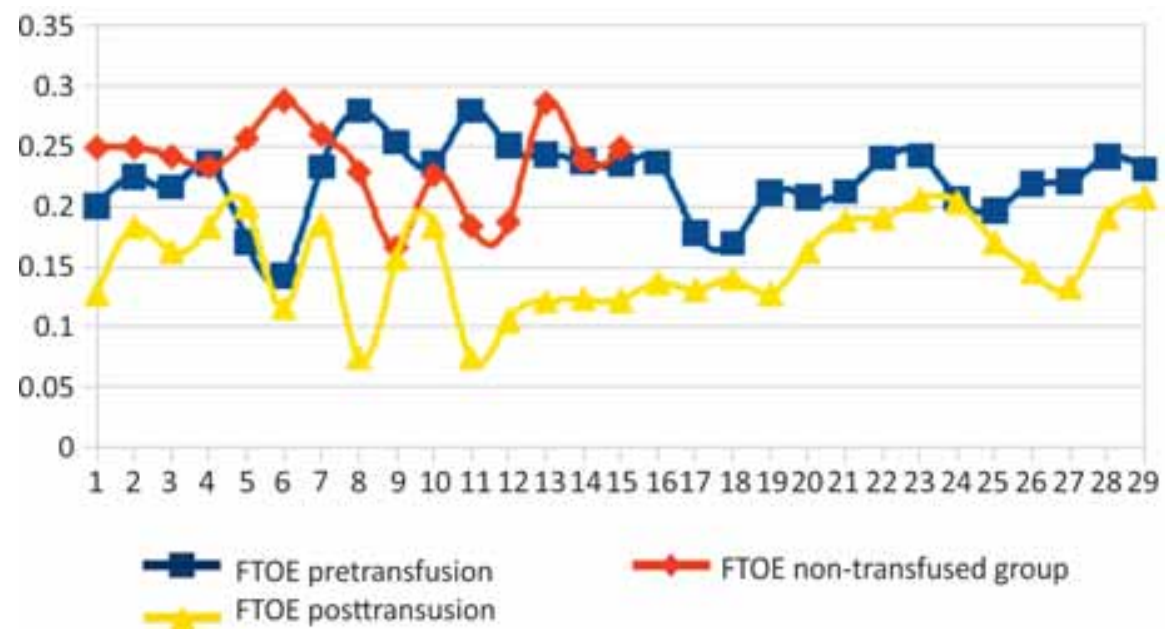

FIGURE 2. Fractiona tissular oxyven extraction (FTOE)

oxygenation measurements such as pulsatile saturation in capillary oxygen ( $\mathrm{SpO} 2)$, tissue perfusion index, lactate $(15,16)$. Since hemoglobin is the main determinant of the oxygen supply, these findings support the argument that, while oxygen release can become limited (anemia), measurable changes in oxygen extraction occur at the tissue level before the actual clinical signs (17). Under these conditions, FTOE may be a more sensitive oxygen measurement. In a prospective study in 2002 that attempted the use of FTOE to identify the need for transfusion (18), oxygen extraction decreased (i.e. it improved) after PRBC transfusion for each clinical indications.

In our study we did not notice changes in tissue metabolism ( $\mathrm{pH}$, lactic acid) in anemic preterm infants, nor changes in tissue perfusion index before or after transfusion. Serum lactate concentration has highly been extensively used in preterm infants as a criteria for PRBC transfusion in anemia, and strongly correlated with transfusion outcome (19).

Because we studied the effects of anemia and transfusion effects in hemodynamically stable preterm infants, metabolic effects were not noticed due to the existence of a sufficient reserve capacity to meet metabolic demands. It should be noted that serum lactate does not always correlate with tissue perfusion. Liver is the main organ that metabolizes excess lactic acid produced at the tissues level. There are transient disorders of aminoacid metabolism in preterm infants which can lead to different levels of lactic acid, independently from the tissue perfusion (20). A limitation of our study is that, although we examined a highly selected, homogeneous stable group, a larger would be needed to track other tissue oxygenation sites (splanchnic, renal) in 
parallel, that could provide more confidence in the immediate clinical relevance of our outcomes. Thus, this analysis is useful for the hypothesis that using either FTOE or rSO2, transfusion thresholds can be established more accurately based on a more direct assessment of tissue oxygenation. In summary, this study shows that PRBC transfusion in stable preterm infants results in a transient increase of $\mathrm{CrSO} 2$ and transient decrease of FTOE, and no change in the $\mathrm{pH}$, serum lactate or systemic oxygen saturation. Our data support the use of NIRS derived measurements (FTOE) in identifying the subclinical imbalance in $\mathrm{O} 2$ delivery and consumption as an objective measurement in anemic preterm infants.

\section{CONCLUSIONS}

We can use the fractional tissue oxygen extraction - FTOE - as indicator of the need for transfusion, along with the value of the hematocrit and the clinical signs. In our study, a fractional oxygen extraction (C-FTOE) pre-transfusion $>0.25$ associated with clinical signs of anemia showed that transfusion was beneficial.

To include NIRS derived measurements in transfusion guidelines, more multicenter studies are required to establish with more accuracy the FTOE limit above is used in anemic preterm infants.

\section{Conflict of interest: none declared Financial support: none declared}

\section{REFERENCES}

1. Sood B.G., Cortez J., McLaughlin K.L., Gupta M. et al. Near infrared spectroscopy as a biomarker for necrotizing enterocolitis following red blood cell transfusion. J Near InfraRedSpectrosc 2014; 22(6):375-388

2. Van Hoften J.C., Verhagen E.A., Keating P. et al. Cerebral tissue oxygen saturation and extraction in preterm infants before and after blood transfusion. Archs Dis Childh Fetal Neonatal Ed 2010; 95:F352-8

3. Wardle S.P., Garr R., Yoxall C.W. A pilot randomised controlled trial of peripheral fractional oxygen extraction to guide blood transfusions in preterm infants. Archs Dis Childh Fetal Neonatal Ed 2002; 86:F22-7.

4. Bailey S.M., Hendricks-Munoz K.D., Mally P. Splanchnic cerebral oxygenation ratio as a marker of preterm infant blood transfusion needs. Transfusion 2012; 52:252-260.

5. Abu Jawdeh E.G., Martin R.J., Dick T.E. et al. The effect of red blood cell transfusion on intermittent hypoxemia in ELBW Infants. J Perinatol 2014; 34: 921-925.

6. Seidel D., Blaser A., Gebauer C., Pulzer F. et al. Changes in regional tissue oxygenation saturation and desaturations after red blood cell transfusion in preterm infants. J Perinatol 2013; 33:282-7.

7. Beena G. Sood, Kathleen McLaughlin, Josef Cortez. Near-infrared spectroscopy: Applications in neonates. Seminars in Fetal \& Neonatal Medicine 20 (2015) 164-72

8. Bailey S., Hendricks-Munoz K., Wells J. Packed red blood cell transfusion increases regional cerebral and splanchnic tissue oxygen saturation in anemic symptomatic preterm infants. Am J Perinat 2010; 27:445-53.

9. Dani C., Pratesi S., Fontanelli G. et al. Blood transfusions increase cerebral, splanchnic, and renal oxygenation in anemic preterm infants. Transfusion 2010; 50: 1220-6.

10. Kussman B.D., Benni P.B., Bergersen L.T. et al. Validation of the FORE-SIGHT ELITE tissue oximeter for measurement of somatic oxygenation in children. Presented at the $37^{\text {th }}$ Annual Meeting of the Society of Cardiovascular Anesthesiologists (SCA Abstract \#97; April 2015, Washington DC).

11. Sorensen L.C., Greisen G. The brains of very preterm newborns in clinically stable condition may be hyperoxygenated. Pediatrics 2009; 124:958-63.

12. Tina L.G., Frigiola A., Abella R., Artale B. et al. Near infrared spectroscopy in healthy preterm and term newborns: correlation with gestational age and standard monitoring parameters. Curr Neurovasc Res 2009; 6(3):148-54.

13. Takami T., Sunohara D., Kondo A., Mizukaki N. et al. Changes in cerebral perfusion in extremely LBW infants during the first $72 \mathrm{~h}$ after birth. Pediatr Res 2010; 68(5):435-9.

14. Frank van Bel, Petra Lemmers, Gunnar Naulaers. Monitoring Neonatal Regional Cerebral Oxygen Saturation in Clinical Practice: Value and Pitfalls, Neonatology 2008; 94:237-244

15. Alkalay A.L., Galvis S., Ferry D.A., Simmons C.F., Krueger R.C. Jr. Hemodynamic changes in anemic premature infants: Are we allowing the hematocrits to fall too low? Pediatrics 2003; 112:838-45.

16. Bohler T., Janecke A., Linderkamp O. Blood transfusion in late anemia of prematurity: Effect on oxygen consumption, heart rate, and weight gain in otherwise healthy infants. Infusions- therapie und Transfusionsmedizin 1994; 21:376-9.

17. J.P. Mintzer, B. Parvez, M. Chelala, G. Alpan et al. Monitoring regional tissue oxygen extraction in neonates $<1250 \mathrm{~g}$ helps identify transfusion thresholds independent of hematocrit J Neonatal Perinatal Med. 2014 Jan 1; 7(2):89-100

18. Wardle S.P., Garr R., Yoxall C.W., Weindling A.M. A pilot randomised controlled trial of peripheral fractional oxygen extraction to guide blood transfusions in preterm infants. Arch Dis Child-Fetal 2002; 86:F22-7.

19. Izraeli S., Ben-Sira L., Harell D., Naor N., Ballin A., Davidson S. Lactic acid as a predictor for erythrocyte transfusion in healthy preterm infants with anemia of prematurity. J Pediatr 1993; 122(4): 629-631.

20. Burton B.K. Inborn errors of metabolism in infancy: A guide to diagnosis. Pediatrics 1998; 102(6): 69. 